\title{
Phase matching condition for enhancement of phase sensitivity in quantum metrology
}

\author{
Jing Liu, Xiaoxing Jing, Xiaoguang Wang* \\ Zhejiang Institute of Modern Physics, Department of Physics, Zhejiang University, Hangzhou 310027, China
}

\begin{abstract}
We find a phase matching condition for enhancement of sensitivity in a Mach-Zehnder interferometer illuminated by an arbitrary state in one input port and an odd(even) state in the other port. Under this condition, the Fisher information becomes maximal with respect to the relative phase of two modes and the phase sensitivity is enhanced. For the case with photon losses, we further find that the phase matching condition keeps unchanged with a coherent state and a coherent superposition state as the input states.
\end{abstract}

PACS numbers: 03.67.-a, 42.50.St, 42.50.Dv

Introduction.-With the development of quantum information theory [1 [5] and quantum technology [6 8 ], quantum metrology is becoming more and more practical nowadays. Improving the precision of a parameter is always the basic theme in quantum metrology. The fundamental procedure of it is the parametrization process. There are three mainly methods to perform this procedure: (i) unitary parametrization; (ii) channel parametrization; (iii) accelerating parametrization. Unitary parametrization is the most useful and well studied method because it is widely applied in the phase estimation, the main task in quantum metrology. The precision measurement of gravity, temperature, week magnetic strength and many other parameters can be classified in the category of phase estimation. Channel parametrization is also widely studied in the channel estimation [9, 10] for many years. With the help of the quantum technology, it may be possible to proceed the parametrization in a accelerating way [11], in which the relativistic effects cannot be neglected any more. In this paper, we mainly focus on the category of phase estimation and study how to effectively enhance the phase sensitivity.

In 1981, Caves [12] found out that, for phase estimation, taking a high intensity coherent state and a low intensity squeezed vacuum state as the input states of a Mach-Zehnder interferometer, the precision can beat the shot-noise limit(quantum standard limit), i.e., $1 / \sqrt{N}$, where $N$ is the total photon number in both modes. Since then, many protocols have been proposed to fulfill the similar job, such as NOON state, entangled coherent state [13], two-mode squeezed state [14], number squeezed states [15] and so on. With high intensity, some of these states can even theoretically achieve or surpass the Heisenberg limit, i.e., $1 / N$. In the pioneer work of Caves [12], to enhance the precision, the phases of the two input states need to satisfy a relation. This can be considered as a kind of phase matching condition (PMC). Thus, it is reasonable to study if there is a more general PMC for more general states to to enhance the phase sensitivity. This is the major motivation of this paper. To depict the precision of a parameter $\theta$, quantum Fisher information(QFI) is an available useful concept because it describes the lower bound on the variance of the estimator $\hat{\theta}$ due to the Cramér-Rao theorem: $\operatorname{Var}(\hat{\theta}) \geq 1 /(\nu F),[16,17]$ where $\operatorname{Var}(\cdot)$ is the variance, $\nu$ is the number of repeated experiments and $F$ is the QFI.

In this paper, we discuss a general scenario of a MachZehnder interferometer. In this interferometer, one of the input port is an arbitrary state and the other one is an even (odd) state. This scenario covers many important cases including the famous protocol of Caves 12], and a recent one proposed by Pezzé and Smerzi [15]. We give an analytic expression of the QFI and identify a PMC to optimize the parameter precision. Under this condition, the QFI is only determined the the average photon numbers of the two modes and the corresponding expectation values of the square of annihilation operators. We then give two examples of our scenario. Further, for the case that photon losses occur in both arms with the same transmission coefficients, and the input state is a product of a coherent state and a coherent superposition state, the analytic expression of QFI is provided. Based on this expression, we prove that the PMC keeps unchanged for

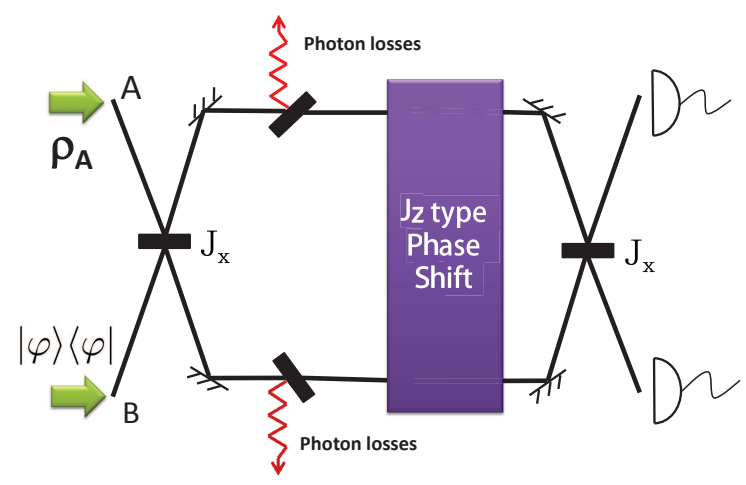

Figure 1: (Color online) Sketch of the Mach-Zehnder interferometer. The transformation of the beam splitters are described by $\exp \left( \pm i \pi J_{x} / 2\right)$, and that of the phase shift is described by $\exp \left(i \theta J_{z}\right)$. The input states in port $\mathrm{A}$ and $\mathrm{B}$ are an arbitrary state and an even(odd) state, respectively. 
any transmission coefficients.

Mach-Zehnder interferometer.-Mach-Zehnder(MZ) interferometer is a well known optical device in quantum metrology which is constructed with two beam splitters and one or two phase shifts. The interferometer we consider here is constructed with two 50:50 beam splitters and two phase shifts in both arms, as shown in Fig. 1. The well used 50:50 beam splitter can be described by [19 21] $B_{x}=\exp \left(-i \frac{\pi}{2} J_{x}\right)$, where $J_{x}=\frac{1}{2}\left(a^{\dagger} b+b^{\dagger} a\right)$ is one of the operators in the Schwinger representation of bosons. The others are $J_{y}=\frac{1}{2 i}\left(a^{\dagger} b-b^{\dagger} a\right)$ and $J_{z}=\frac{1}{2}\left(a^{\dagger} a-b^{\dagger} b\right)$. Here $a, b$ are annihilation operators for ports $\mathrm{A}$ and $\mathrm{B}$, respectively. Operators $J_{x}, J_{y}, J_{z}$ satisfy the commutation: $\left[J_{i}, J_{j}\right]=i \epsilon_{i j k} J_{k}$, with $\epsilon_{i j k}$ the so-called Levi-Civita symbol. Usually, a phase shift can be expressed by a unitary transformation $\exp \left[i \theta \hat{N}_{\mathrm{A}(\mathrm{B})}\right]$, where $\hat{N}_{\mathrm{A}}=a^{\dagger} a, \hat{N}_{\mathrm{B}}=b^{\dagger} b$ and $\theta$ is an unknown parameter. In our scenario, denoting $\theta$ as the relative phase between the two arms, one can describe the transformation of the total phase shift by the operator $P_{z}=\exp \left(i \theta J_{z}\right)$. With above devices we can construct a well used MZ interferometer, the transformation of which can be written as $U_{\mathrm{mz}}=B_{x} P_{z} B_{x}^{\dagger}$. Through some algebra, one can find that this transformation can be simplified as

$$
U_{\mathrm{MZ}}=\exp \left(-i \theta J_{y}\right),
$$

which is a rotation along $y$ direction.

Phase matching condition for QFI.-Quantum Fisher information(QFI) is a central concept in quantum metrology, and it is defined as [16, 17] $F:=\operatorname{Tr}\left(\rho L^{2}\right)$, where $L$ is the so-called symmetric logarithmic derivative determined by $\partial_{\theta} \rho_{\theta}=\left(\rho_{\theta} L+L \rho_{\theta}\right) / 2$. We consider a separable input state $\rho_{\text {in }}=\rho_{\mathrm{A}} \otimes \rho_{\mathrm{B}}$. Here $\rho_{\mathrm{A}}$ is an arbitrary state and $\rho_{\mathrm{B}}=|\varphi\rangle\langle\varphi|$ is an even(odd) state. Utilizing the spectral decomposition $\rho_{\mathrm{A}}=\sum_{j=1}^{M} p_{j}\left|\psi_{j}\right\rangle\left\langle\psi_{j}\right|$, the quantum Fisher information can be written as [16 18$]$

$$
F=\sum_{j=1}^{M} 4 p_{j}\left\langle\phi_{j}\left|J_{y}^{2}\right| \phi_{j}\right\rangle-\sum_{j, j^{\prime}=1}^{M} \frac{8 p_{j} p_{j^{\prime}}}{p_{j}+p_{j^{\prime}}}\left|\left\langle\phi_{j}\left|J_{y}\right| \phi_{j^{\prime}}\right\rangle\right|^{2},
$$

where $M$ is the dimension of the support of $\rho_{\mathrm{A}}$ and $\left|\phi_{j}\right\rangle=$ $\left|\psi_{j}\right\rangle \otimes|\varphi\rangle$ is an eigenstate of $\rho_{\text {in }}$ with eigenvalue $p_{j}$.

As the input state in port B is an even(odd) state in our scenario, it satisfies $\langle\varphi|b| \varphi\rangle=0$. Then after some algebra, the QFI can be expressed by

$$
F=2 \bar{n}_{\mathrm{A}} \bar{n}_{\mathrm{B}}+\bar{n}_{\mathrm{A}}+\bar{n}_{\mathrm{B}}-2 \operatorname{Re}\left(\left\langle a^{\dagger 2}\right\rangle\left\langle b^{2}\right\rangle\right),
$$

where $\bar{n}_{\mathrm{A}}=\operatorname{Tr}\left(\rho_{\mathrm{A}} a^{\dagger} a\right)$ and $\bar{n}_{\mathrm{B}}=\left\langle\varphi\left|b^{\dagger} b\right| \varphi\right\rangle$ are average photon numbers for mode $a$ and $b$, respectively. To optimize this Fisher information, the input states need to satisfy the following PMC:

$$
\left|\operatorname{Arg}\left(\left\langle a^{2}\right\rangle\right)-\operatorname{Arg}\left(\left\langle b^{2}\right\rangle\right)\right|=\pi .
$$

Under this condition, the QFI becomes

$$
F_{\mathrm{m}}=2 \bar{n}_{\mathrm{A}} \bar{n}_{\mathrm{B}}+\bar{n}_{\mathrm{A}}+\bar{n}_{\mathrm{B}}+2\left|\left\langle a^{2}\right\rangle\right|\left|\left\langle b^{2}\right\rangle\right| .
$$

One key feature of the above result is that the QFI is only determined by the mean photon numbers and the expectations of $a^{2}$ and $b^{2}$. We emphasize that one input state is arbitrary.

For the case that $\left|\left\langle a^{2}\right\rangle\right|\left|\left\langle b^{2}\right\rangle\right|=0$, the phases of the input states can be chosen arbitrarily. For example, if $\rho_{\mathrm{B}}$ is a Fock state, i.e., $\rho_{\mathrm{B}}=|n\rangle\langle n|$, then the QFI reduces to $F_{\mathrm{m}}=2 \bar{n}_{\mathrm{A}} \bar{n}_{\mathrm{B}}+\bar{n}_{\mathrm{A}}+\bar{n}_{\mathrm{B}}$, which is the result in Ref. [15] and has been discussed in detail. In the following, we give two examples of the PMC.

Example 1.-In this example, we choose $\rho_{\mathrm{A}}$ be a coherent state $|\beta\rangle\langle\beta|$ and $\rho_{\mathrm{B}}$ a coherent superposition state $|\alpha\rangle_{+}\langle\alpha|$, where $|\alpha\rangle_{+}=N_{\alpha}(|\alpha\rangle+|-\alpha\rangle)$ with $|\alpha\rangle$ also a coherent state and $N_{\alpha}^{2}=1 /\left(2+2 e^{-2|\alpha|^{2}}\right)$. It is easy to find that ${ }_{+}\langle\alpha|b| \alpha\rangle_{+}=0$. Here, we denote $\alpha=|\alpha| \exp \left(i \Phi_{\alpha}\right)$, $\beta=|\beta| \exp \left(i \Phi_{\beta}\right)$, then from Eq. (4), the phase matching condition can be specifically written as

$$
\left|\Phi_{\alpha}-\Phi_{\beta}\right|=\frac{\pi}{2} .
$$

Under this condition, the QFI reduces to

$$
F_{\mathrm{m}}=2 \bar{n}_{\mathrm{A}} \bar{n}_{\mathrm{B}}+\bar{n}_{\mathrm{A}}+\bar{n}_{\mathrm{B}}+2 \bar{n}_{\mathrm{A}}|\alpha|^{2},
$$

where $\bar{n}_{\mathrm{A}}=|\beta|^{2}$ and $\bar{n}_{\mathrm{B}}=|\alpha|^{2} \tanh |\alpha|^{2}$. As $\tanh |\alpha|^{2}$ is a monotonic function and very close to 1 for $|\alpha| \geq 2$, then for most value of $|\alpha|, \bar{n}_{\mathrm{B}}$ is equal to $|\alpha|^{2}$, and the QFI reduces to $F_{\mathrm{m}}=4 \bar{n}_{\mathrm{A}} \bar{n}_{\mathrm{B}}+\bar{n}_{\mathrm{A}}+\bar{n}_{\mathrm{B}}$. It is not difficult to obtain that $F_{\mathrm{m}} \leq N^{2}+N$, where $N=\bar{n}_{\mathrm{A}}+\bar{n}_{\mathrm{B}}$ is the total photon number. The equality can be achieved when $\bar{n}_{\mathrm{A}}=$ $\bar{n}_{\mathrm{B}}$. For the case that total photon number $N$ is fixed, this bound is the optimal value of the QFI. Considering the PMC, the optimal value can be achieved when $\beta=$ $\pm i \alpha$. From the optimal value of $F_{\mathrm{m}}=N^{2}+N \geq N^{2}$, one can find that, with high intensity, the pair of the coherent state $| \pm i \alpha\rangle\langle \pm i \alpha|$ and the coherent superposition state $|\alpha\rangle_{+}$can surpass the Heisenberg limit. Ref. [13] considered the same input states in our scenario but with only one phase shift in one arm. In their case, utilizing the similar analysis above, it is easy to find that the input states $|\alpha\rangle$ and $|\alpha\rangle_{+}$is the optimal choice and can reach the maximum value of QFI.

Example 2.-Another well known even state is the squeezed vacuum state, which is defined as [22] $|\xi\rangle=$ $S(\xi)|0\rangle$, where the squeezing operator reads $S(\xi)=$ $\exp \left(\frac{1}{2} \xi^{*} b^{2}-\frac{1}{2} \xi b^{\dagger^{2}}\right)$ with $\xi=|\xi| \exp \left(i \Phi_{\xi}\right)$. For convenience, we still choose the input state in port $\mathrm{A}$ as a coherent state $|\beta\rangle\langle\beta|$. In this example, the PMC is

$$
2 \Phi_{\beta}-\Phi_{\xi}=0 .
$$

Under this condition, the QFI can be expressed as

$$
F_{\mathrm{m}}=2 \bar{n}_{\mathrm{A}} \bar{n}_{\mathrm{B}}+\bar{n}_{\mathrm{A}}+\bar{n}_{\mathrm{B}}+2 \bar{n}_{\mathrm{A}} \sqrt{\bar{n}_{\mathrm{B}}^{2}+\bar{n}_{\mathrm{B}}},
$$

where $\bar{n}_{\mathrm{A}}=|\beta|^{2}$ and $\bar{n}_{\mathrm{B}}=\sinh ^{2}|\xi|$. This equation is equivalent to the corresponding equation in Ref. [23]. In 


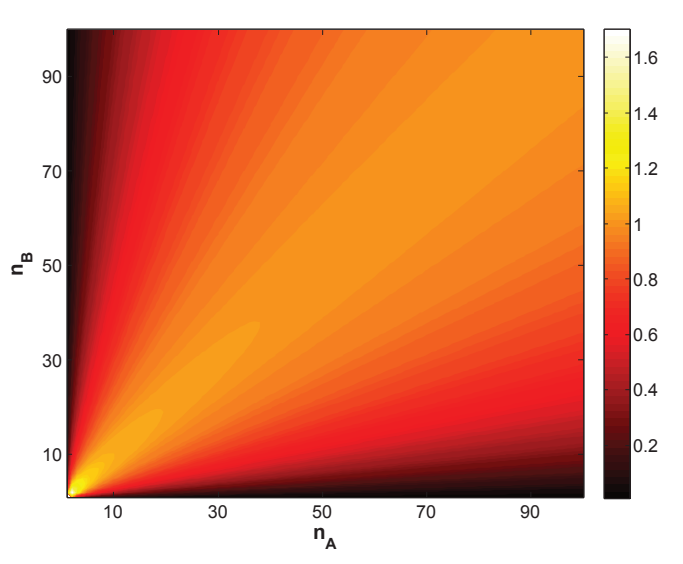

Figure 2: (Color online) The variation of $F_{\mathrm{m}} / N^{2}$ with the average particle number $\bar{n}_{\mathrm{A}}$ and $\bar{n}_{\mathrm{B}}$. The input states here are a coherent state $|\beta\rangle\langle\beta|$ in port $\mathrm{A}$ and a squeezed vacuum state $|\xi\rangle\langle\xi|$ in port $\mathrm{B}$.

this expression of QFI, it is only related to the average photon numbers in both ports: $\bar{n}_{\mathrm{A}}$ and $\bar{n}_{\mathrm{B}}$. Figure 2 shows the variation of $F_{\mathrm{m}} / N^{2}$ with the change of $\bar{n}_{\mathrm{A}}$, $\bar{n}_{\mathrm{B}}$. From this plot one can find that the optimal value of quantum Fisher information for a fixed $N$ is obtained near the $n_{\mathrm{A}}=n_{\mathrm{B}}$ line, especially for a large $N$. This is because when $\bar{n}_{\mathrm{B}}$ is large, for fixed $N, F_{\mathrm{m}} \simeq 4 \bar{n}_{\mathrm{A}} \bar{n}_{\mathrm{B}}+$ $\bar{n}_{\mathrm{A}}+\bar{n}_{\mathrm{B}} \leq N^{2}+N$ and the optimal value can be achieved when $\bar{n}_{\mathrm{A}}=\bar{n}_{\mathrm{B}}$. Adding the PMC, the optimal choice for a large $N$ is that $\beta=\exp \left(i \Phi_{\xi} / 2\right) \sinh |\xi|$. Also, from this figure one can see that with the increase of $N$, the range that $F_{\mathrm{m}}>N^{2}$ is increasing, which indicates that high intensity input state is good for the enhancement of the phase sensitivity.

$P M C$ with unbanlanced beam splitter.-For a more general beam splitter transformation $\exp \left(i \tau J_{x}\right)$ with $\tau \in$ $[0,2 \pi)$, the total setup of the interferometer can be described by

$$
U_{\mathrm{MZ}}=\exp \left[i \theta\left(J_{z} \cos \tau-J_{y} \sin \tau\right)\right] .
$$

If we restrict the input state $\rho_{\mathrm{A}}$ as a pure state, i.e., $|\psi\rangle\langle\psi|$, and $\rho_{\mathrm{B}}$ is still an even(odd) state, the QFI reads $F=4 \operatorname{Var}\left(J_{z} \cos \tau-J_{y} \sin \tau\right)$. Due to the property of even(odd) state, one can find that

$$
F=4 \cos ^{2} \tau \operatorname{Var}\left(J_{z}\right)+4 \sin ^{2} \tau \operatorname{Var}\left(J_{y}\right) .
$$

It is known that for product states

$$
\operatorname{Var}\left(J_{z}\right)=\frac{1}{4}\left[\operatorname{Var}\left(a^{\dagger} a\right)+\operatorname{Var}\left(b^{\dagger} b\right)\right],
$$

and $\operatorname{Var}\left(a^{\dagger} a\right), \operatorname{Var}\left(b^{\dagger} b\right)$ are irrelevant to the phase, therefore, the phase matching condition is decided by the second item $\operatorname{Var}\left(J_{y}\right)$ and keeps the same form with Eq. (4).

$P M C$ with photon losses.-Now we consider the effects of photon losses on the PMC. The scenario we use is shown in Fig. 1. Traditionally, the photon losses can be described by a fictitious beam splitter [24 29]. Here we use $B^{T}=\exp \left[i(2 \arccos \sqrt{T}) J_{x}\right]$ to describe this fictitious beam splitter. $T$ is the so-called transmission coefficient and we also define $R=1-T$ as the reflection coefficient. When $T=1(R=0)$, there are no photon losses in the interferometer and when $T=0(R=1)$, all the photons leak out of the interferometer. This total photon loss scenario can be mapped into a neat scenario, which includes two steps: first the input state goes through a particle loss channel, then the output state imports into a MZ interferometer without losses.

We assume that the leak in both arms share the same transmission coefficient $T$ and the input state is separable: $\rho_{\mathrm{A}} \otimes \rho_{\mathrm{B}}$. Then after the particle loss channel, the reduced density matrix $\rho$ reads

$$
\rho=\operatorname{Tr}_{\mathrm{CD}}\left(\Gamma^{\mathrm{D}} \Gamma^{\mathrm{C}} \rho_{\mathrm{A}} \otimes \rho_{\mathrm{B}} \Gamma^{\mathrm{C} \dagger} \Gamma^{\mathrm{D} \dagger}\right),
$$

where the operator of the particle loss channel can be expressed by $\Gamma^{\mathrm{C}}=\exp \left[i \sqrt{2} \arccos \sqrt{T}\left(J_{x}^{\mathrm{AC}}+J_{y}^{\mathrm{BC}}\right)\right]$ and $\Gamma^{\mathrm{D}}=\exp \left[i \sqrt{2} \arccos \sqrt{T}\left(J_{x}^{\mathrm{BD}}+J_{y}^{\mathrm{AD}}\right)\right]$. Here $J_{x}^{\mathrm{AC}}=$ $\frac{1}{2}\left(a^{\dagger} c+a c^{\dagger}\right)$ and $J_{y}^{\mathrm{BC}}=\frac{1}{2 i}\left(b^{\dagger} c-b c^{\dagger}\right)$ with $c, c^{\dagger}$ the annihilation and creation operators of mode C. So as $J_{x}^{\mathrm{BD}}$ and $J_{y}^{\mathrm{AD}}$. Next $\rho$ goes through a usual MZ interferometer, which can be described by $\exp \left(-\theta J_{y}\right)$. Utilizing this representation, this photon losses scenario can be classified into a usual MZ interferometer scenario with a mixed input state.

Now we choose the initial states as $\rho_{\mathrm{A}}=\left|i \alpha e^{i \Phi}\right\rangle\left\langle i \alpha e^{i \Phi}\right|$ and $\rho_{\mathrm{B}}=|\alpha\rangle_{+}\langle\alpha|$. Here $\Phi$ is the relative phase and $\Phi \in[0, \pi)$. Based on the PMC, the optimal value of it is zero for no loss scenario. The similar scenario has been considered with only one phase shift in one arm [13, 30, 31]. Through some calculations, one can obtain the analytic expression of the QFI

$$
\begin{aligned}
F= & 4 T|\alpha|^{2}\left[N_{\alpha}^{2}+T|\alpha|^{2}\left(2 N_{\alpha}^{2}-1\right)\right]+4 T^{2}|\alpha|^{4} \mathcal{G} \cos ^{2} \Phi \\
& -16 T^{2} N_{\alpha}^{4}|\alpha|^{4}\left(1-p_{r}^{2}\right) p_{t}^{2} \sin ^{2} \Phi,
\end{aligned}
$$

where $p_{r}=\exp \left(-2|\alpha|^{2} R\right), p_{t}=\exp \left(-2|\alpha|^{2} T\right)$ and $\mathcal{G}=$ $1-4 N_{\alpha}^{4}\left(1-p_{r}^{2}\right)$. From this equation, one can see that for a fixed $\alpha$ and transmission coefficient $T$, the maximum value of Eq. (14) can always be obtained at $\Phi=0$, which indicates that the phase matching condition remains unchanged in this photon losses case and is not effected by the transmission coefficient $T$. Under the phase matching condition, the quantum Fisher information reads

$$
F_{\mathrm{m}}=4 T N_{\alpha}^{2}|\alpha|^{2}+8 T^{2} N_{\alpha}^{2}|\alpha|^{4}\left[1-2 N_{\alpha}^{2}\left(1-p_{r}^{2}\right)\right] .
$$

Utilizing the input average photon number $\bar{n}_{\mathrm{A}}$ and the input total photon number $N$, it can be rewritten as

$$
F_{\mathrm{m}}=T N+2 T^{2} N \bar{n}_{\mathrm{A}}\left[1-2 N_{\alpha}^{2}\left(1-p_{r}^{2}\right)\right] .
$$


This equation can reduce to Eq. (7) for $T=1(R=0)$. From this equation, one can find that the photon losses have a negative influence on the QFI. With the decrease of the transmission coefficient $T$, the QFI reduces. For a small losses, namely $R$ is very small, the QFI reduces to

$$
F_{\mathrm{m}}=N+2 N \bar{n}_{\mathrm{A}}-\left[1+4 \bar{n}_{\mathrm{A}}(N+1)\right] N R
$$

When $R<R_{c}$, with $R_{c}=2 \bar{n}_{\mathrm{A}} /\left[1+4 \bar{n}_{\mathrm{A}}(N+1)\right]$, the QFI can still larger than $N$, which indicates that these input states can still surpass the shot-noise limit. This device is loss-tolerant and robust within the region that $R \in\left[0, R_{c}\right]$. For a large $N, R_{c} \simeq 1 /(2 N)$.

Conclusion.-In summary, we have considered a general scenario of a Mach-Zehnder interferometer, where the input state in one port of the interferometer is an arbitrary state and the other one is an even(odd) state. We have provided an analytic expression of the QFI and showed a general PMC under which the phase sensitivity can be enhanced. We give two explicit examples with one port in a coherent state and another in a even coherent state or the squeezed vacuum state. The PMC plays an important role to improve the sensitivity in these cases.

For the unbalanced beam splitters, we find the PMC is unchanged with one port in a arbitrary pure stare and another in an even(odd) state. We also considered the example of coherent superposition state with photon losses and find that the PMC keeps unchanged and is not affected by the transmission coefficients. Besides, for a small loss, we show that this setup is robust and loss-tolerant. The present work sheds new light on the problem of how to enhance phase sensitivity in quantum metrology.

Acknowledgments.-This work was supported by the NFRPC through Grant No. 2012CB921602, the NSFC through Grants No. 11025527 and No. 10935010.

* Electronic address: xgwang@zimp.zju.edu.cn

[1] V. Giovannetti, S. Lloyd, and L. Maccone, Nat. Photonics 5, 222 (2011); V. Giovannetti, S. Lloyd and L. Maccone, Phys. Rev. Lett. 96, 010401 (2006); C. C. Gerry and J. Mimih, Contemporary Physics, 51, 497-511 (2010).

[2] M. G. Genoni, S. Olivares, and M. G. A. Paris, Phys. Rev. Lett. 106, 153603 (2011); L. Pezzé and A. Smerzi, Phys. Rev. Lett. 100, 073601 (2008); Y. C. Liu, G. R. Jin and L. You, Phys. Rev. A 82, 045601 (2010); F. Benatti, R. Floreanini and U. Marzolino, J. Phys. B: At. Mol. Opt. Phys. 44, 091001 (2011); C. Gagatsos, O. Oreshkov and N. Cerf, Phys. Rev. A 87, 042307 (2013).

[3] K. P. Seshadreesan, P. M. Anisimov, H. Lee, and J. P. Dowling, New J. Phys. 13, 083026 (2011); D. W. Berry, B. L. Higgins, S. D. Bartlett, M. W. Mitchell, G. J. Pryde, and H. M. Wiseman, Phys. Rev. A 80, 052114 (2009); R. Birrittella, J. Mimih and C. C. Gerry, Phys. Rev. A 86, 063828 (2012).
[4] C. C. Gerry and A. Benmoussa, Phys. Rev. A 65, 033822 (2002); C. C. Gerry and R. A. Campos, Phys. Rev. A 64, 063814 (2001); B. M. Escher, R. L. de MatosFilho, and L. Davidovich, Nat. Phys. 7, 406 (2011);

[5] X.-M. Lu, S. Luo and C. H. Oh, Phys. Rev. A 86, 022342 (2012); X.-M. Lu, Z. Sun, X. Wang, S. Luo, and C. H. Oh, Phys. Rev. A 87050302 (2013); G. Tóth and D. Petz, Phys. Rev. A 87, 032324 (2013).

[6] J. Estève, C. Gross, A. Weller, S. Giovanazzi, and M. K. Oberthaler, Nature (London) 455, 1216 (2008); M. F. Riedel, P. Böhi, Y. Li, T. W. Hänsch, A. Sinatra, and P. Treutlein, Nature (London) 464, 1170 (2010).

[7] J. Appel, P. J. Windpassinger, D. Oblak, U. B. Hoff, N. Kjaegaard, and E. S. Polzik, Proc. Natl. Acad. Sci. U.S.A. 106, 10960 (2009).

[8] T. Nagata, R. Okamoto, J. L. O'Brien, K. Sasaki, and S. Takeuchi, Science 316, 726 (2007).

[9] A. Fujiwara, Phys. Rev. A 63, 042304 (2001); A. Fujiwara, Phys. Rev. A 65, 012316 (2001); H. Imaiand A. Fujiwara, J. Phys. A 40, 4391 (2007); A. Fujiwara and H. Imai, J. Phys. A 36, 8093 (2003).

[10] A. Monras and M. G. A. Paris, Phys. Rev. Lett. 98, 160401 (2007); D. Collins, Phys. Rev A 87, 032301 (2013); J. Kahn, Phys. Rev. A 75, 022326 (2007); M. Hotta and T. Karasawa, Phys. Rev A 78, 012332 (2008) and so on.

[11] M. Ahmadi, D. E. Bruschi, N. Friis, C. Sabn, G. Adesso and I. Fuentes, e-print: arXiv:1307.7082 [quant-ph].

[12] C. M. Caves, Phys. Rev. D 23, 1693-1708 (1981).

[13] J. Joo, W. J. Munro and T. P. Spiller, Phys. Rev. Lett. 107, 083601 (2011).

[14] P. M. Anisimov, G. M. Raterman, A. Chiruvelli, W. N. Plick, S. D. Huver, H. Lee and J. P. Dowling, Phys. Rev. Lett. 104, 103602 (2010).

[15] L. Pezzé and A. Smerzi, Phys. Rev. Lett. 110, 163604 (2013).

[16] C. W. Helstrom, Quantum Detection and Estimation Theory (Academic, New York, 1976).

[17] A. S. Holevo, Probabilistic and Statistical Aspects of Quantum Theory (NorthHolland, Amsterdam, 1982).

[18] J. Liu, X. Jing, W. Zhong and X. Wang, submitted.

[19] B. Yurke, S. L. McCall and J. R. Klauder, Phys. Rev. A 33, 4033 (1986).

[20] R. A. Campos, B. E. A. Saleh and M. C. Teich, Phys. Rev. A 40, 1371 (1989).

[21] B.C. Sanders and G. J. Milburn, Phys. Rev. Lett. 75, 2944-2947 (1995).

[22] M. O. Scully and M. S. Zubairy, Quantum Optics (Cambridge University Press, Cambridge, 1997).

[23] M. Jarzyna and R. Demkowicz-Dobrzański, Phys. Rev. A 85, 011801(R) (2012).

[24] R. Demkowicz-Dobrzanski, U. Dorner, B. J. Smith, J. S. Lundeen, W. Wasilewski, K. Banaszek, and I. A. Walmsley, Phys. Rev. A 80, 013825 (2009).

[25] S. D. Huver, C. F. Wildfeuer, and J. P. Dowling, Phys. Rev. A 78, 063828 (2008).

[26] U. Dorner, R. Demkowicz-Dobrzanski, B. J. Smith, J. S. Lundeen, W. Wasilewski, K. Banaszek, and I. A. Walmsley, Phys. Rev. Lett. 102, 040403 (2009).

[27] T. W. Lee, S. D. Huver, H. Lee, L. Kaplan, S. B. McCracken, C. Min, D. B. Uskov, C. F. Wildfeuer, G. Veronis, and J. P. Dowling, Phys. Rev. A 80, 063803 (2009).

[28] N. Gkortsilas, J. J. Cooper, and J. A. Dunningham, Phys. Rev. A 85, 063827 (2012). 
[29] X.-X. Zhang, Y.-X. Yang, and X.-B. Wang, Phys. Rev. A 88, 013838 (2013).

[30] Y. M. Zhang, X. W. Li, W. Yang and G. R. Jin, e-print:
arXiv:1307.7353

[31] X. Jing, J. Liu, W. Zhong and X. Wang, e-print: arXiv:1307.8009 